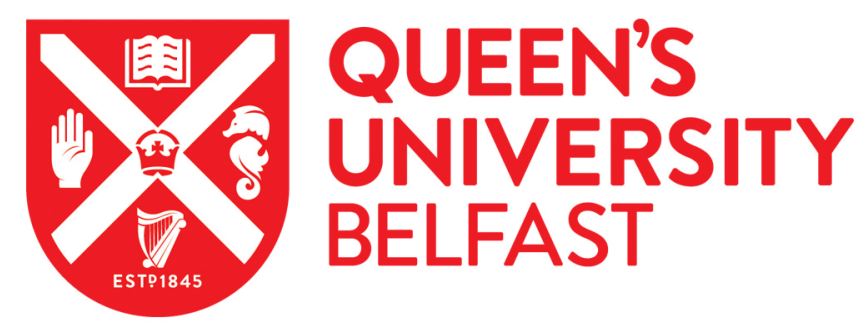

\title{
Serum profiling identifies ibrutinib as a treatment option for young adults with B-cell acute lymphoblastic leukaemia
}

Jordaens, S., Cooksey, L., Bonney, S., Orchard, L., Coutinho, M., Van Tendeloo, V., Mills, K. I., Orchard, K., \& Guinn, B-A. (2020). Serum profiling identifies ibrutinib as a treatment option for young adults with B-cell acute lymphoblastic leukaemia. British Journal of Haematology, 189(3), 500-512. https://doi.org/10.1111/bjh.16407

\section{Published in:}

British Journal of Haematology

\section{Document Version:}

Peer reviewed version

Queen's University Belfast - Research Portal:

Link to publication record in Queen's University Belfast Research Portal

\section{Publisher rights}

(c) 2020 British Society for Haematology and John Wiley \& Sons Ltd.

This work is made available online in accordance with the publisher's policies. Please refer to any applicable terms of use of the publisher.

\section{General rights}

Copyright for the publications made accessible via the Queen's University Belfast Research Portal is retained by the author(s) and / or other copyright owners and it is a condition of accessing these publications that users recognise and abide by the legal requirements associated with these rights.

\section{Take down policy}

The Research Portal is Queen's institutional repository that provides access to Queen's research output. Every effort has been made to ensure that content in the Research Portal does not infringe any person's rights, or applicable UK laws. If you discover content in the Research Portal that you believe breaches copyright or violates any law, please contact openaccess@qub.ac.uk. 


\section{Sero-profiling identifies new targets for the treatment of adults with B-cell acute lymphoblastic leukaemia}

Stephanie Jordaens ${ }^{1,2 \dagger}$, Leah Cooksey ${ }^{1 \dagger}$, Stephanie Bonney ${ }^{3}$, Laurence Orchard ${ }^{3}$, Matthew Coutinho $^{1}$, Viggo Van Tendeloo ${ }^{2}$, Ken I. Mills ${ }^{4}$, Kim Orchard ${ }^{5} \&$ Barbara-ann Guinn ${ }^{1,3}$

${ }^{1}$ Department of Biomedical Sciences, University of Hull, Hull, UK; ${ }^{2}$ Vaccine \& Infectious Disease Institute, Laboratory of Experimental Hematology, University of Antwerp, Belgium; ${ }^{3}$ Cancer Sciences Unit, Somers Cancer Sciences Building, University of Southampton, Southampton; ${ }^{4}$ Centre for Cancer Research and Cell Biology, Queens University Belfast, Lisburn Road, Belfast; ${ }^{5}$ Department of Haematology, University Hospital Southampton NHS Foundation Trust and University of Southampton, Southampton, UK.

$\dagger$ S.J. and L.C. contributed equally to this study

Current address

Keywords: acute lymphoblastic leukaemia, antigen identification, serum profiling, immunotherapy, survivin, bone marrow tyrosine kinase on chromosome X (BMX). 


\begin{abstract}
Acute lymphoblastic leukaemia (ALL) is a haematological malignancy that is characterized by the uncontrolled proliferation of immature lymphocytes. $80 \%$ of cases occur in children where ALL is well understood and treated. However it has a devastating affects on adults, where multi-agent chemotherapy is the standard of care with allogeneic stem cell transplantation for those who are eligible. Immunotherapy has the potential to kill B-ALL lymphoblasts and improve survival rates.

We used sero-profiling to compare thirteen sera samples from nine presentation adult BALL patients with age- and sex-matched healthy volunteer (HV) sera and identified 69 differentially sero-recognised antigens ( $\mathrm{p} \leq 0.02$; fold change $>1.5$ ) including 19 that were preferentially recognised by patients sera. Three antigens bone marrow tyrosine kinase on chromosome $\mathrm{X}$ (BMX), dCTP pyrophosphatase 1 (DCTPP1) and vestigial like 4 (VGLL4) were chosen for further analysis based on existing literature and STRING analysis. BMX plays a crucial role in the Bruton's Tyrosine Kinase (Btk) pathway which is targetted by ibrutinib. In clinical trials for relapsed/refractory B-cell disorders such as chronic lymphocytic leukaemia (CLL) and mantle cell lymphoma, Ibrutinib shows no dose limiting toxicity and high response rates, even as a single agent, suggesting adult BALL would also be a worthy target patient group for future clinical trials using ibrutinib. Each antigen was examined for their association with disease stage, cytogenetics and survival. 12 of 19 showed statistically significantly associations including cyclin G associated kinase (GAK) and TOX high mobility group box family member 2 (TOX2) transcript variant 3 , whose above median expression were associated with 5 -year overall survival.

For the first time, we have used proto-array analysis to identify antigens recognised by antibodies in adult B-ALL sera at disease diagnosis. These antigens have provided novel insights into B-ALL and identified a new target patient population for existing, and seemingly effective, immunotherapy treatment.
\end{abstract}




\section{INTRODUCTION}

B-cell acute lymphoblastic leukaemia (B-ALL) is a clonal malignant disease that is characterised by the accumulation of genetically damaged precursors of $\mathrm{B}$ lineage lymphocytes. This lineage of ALL affects immature B-cells, suppressing normal haematopoiesis and affecting the ability of healthy immature B-cells to differentiate and mature into functional B and plasma cells. Like all leukaemias, ALL rapidly spreads throughout the body making localised treatments of little benefit. In addition, most ALL patients will achieve first remission but experience high relapse rates with long term disease free survival ranging from $15-45 \%^{1}$ depending on patient age.

Immunotherapy is currently used to treat ALL patients through allogeneic haematopoietic stem cell transplant and can improve overall survival for patients to $27-65 \% .^{2-4}$ To boost the graft versus host effect and maximise the chances of successful transplantation of the donor stem cells, patients are given donor leukocyte infusions to support the graft. However, over one third of patients will still relapse and the mortality rates associated with SCTs remain high. Additional immunotherapy strategies for B-ALL include those targeting CD20, a surface marker of B-lineage lymphocytes present in $25 \%$ of patients with pre-B ALL and nearly all mature ALL cells. ${ }^{5}$ Rituximab, a humanized anti-CD20 antibody, ${ }^{6}$ was replaced by second-generation anti-CD20 mAbs, Ofatumumab and Obinutuzumab, to overcome its limitations. ${ }^{5}$ The ubiquitous B-cell marker, CD19 is unsuitable as a target for naked mAbs because it internalizes upon binding ${ }^{5}$ however antibody-drug conjugates including SAR3419, which fuses a humanized anti-CD19 antibody and maytansin and subsequently SGN-CD19A (Denintuzumab) have shown promise. ${ }^{5}$ CD22 is expressed on leukemic blasts in $90 \%$ of patients with pre-B ALL and mature ALL. Inotuzumab ozogamicin is an antibody-drug conjugate developed to target CD22 and couples an engineered humanized monoclonal immunoglobulin G4 antibody against $\mathrm{CD} 22$ conjugated to calicheamicin. ${ }^{5,7}$

Bispecific T cell engagers (also known as BiTEs) conjugate the antigen binding domains of two monoclonal antibodies recognizing leukaemic cells and cytotoxic T cells, a CD3and a CD19-binding part, to direct cytotoxic $\mathrm{T}$ cells against malignant $\mathrm{B}$ cells. Blinatumomab was the first clinically approved bispecific $\mathrm{T}$ cell engagers for use in ALL - reviewed recently by Hathaway et al. ${ }^{8}$ Cellular immunotherapy has gained considerable 
attention with the development of chimeric antigen receptor (CAR) T cells. These are Tcells that have been genetically modified to express a T-cell receptor (TCR) with a specific antigen binding site by fusing a specific monoclonal antibody single-chain variant fragment (scFV), a hinge domain, transmembrane domain and intracellular $\mathrm{T}$ cell activation domains often in combination with costimulatory domains. ${ }^{5,7,9}$ Although, they all differ in design, trials have shown similar positive results ${ }^{5,10-12}$ albeit that on-tumour off-target effects caused by CD19 expression on both normal and malignant Blymphocytes mean that CAR $\mathrm{T}$ cells do not distinguish between tumour and healthy cells. ${ }^{5}$

Despite the potential of existing therapies, new treatments targets are needed to further enhance survival rates for patients with adult B-ALL. These have the potential to improve existing therapeutic strategies through enhanced sensitivity and specificity for adult BALL and identify biomakers to personalised therapies that are applicable to a wider range of patients, and by enhancing our understanding of the disease process provide more effective treatments to enhance patient survival. For the first time we used human protein microrrays (proto-arrays) that contain over 9,000 individally purified human proteins to determine the preferential recognition of antigens by antibodies in adult B-ALL patient sera. This has helped determine which antigens are frequently recognised by patients compared with age- and sex-matched healthy volunteers (HV) samples and as such would make good targets for immunotherapies.

\section{MATERIALS AND METHODS}

Patient samples. Adult B-ALL samples were collected from patients, attending the Departments of Haematology at Southampton University Hospitals Trust, Portsmouth Hospitals NHS Trust and the Royal Devon and Exeter Foundation Trust, following informed consent and local ethical approval (REC 07/H0606/88). Leukaemic blasts, mononuclear cells and sera were isolated from bone marrow or peripheral blood and were collected in EDTA or from clotted blood. Sera and white blood cells were also isolated from age and sex-matched HV samples following informed consent and local ethical approval (LREC 228/02/T), or provided by the North London Blood Transfusion Centre (NLBTC) and all samples were stored at $-80^{\circ} \mathrm{C}$ for later use. Red blood cells were lysed 
using red cell lysis buffer, the cells were washed in phosphate buffered saline (PBS) and either $2.5 \times 10^{4}$ spotted onto two sites on glass slides and allowed to dry for 4-16 h prior to being double wrapped in cling film and stored at $-20^{\circ} \mathrm{C}$ for ICC or snap-frozen as pellets at $10^{5}-10^{6}$ cells per tube at $-80^{\circ} \mathrm{C}$.

Cell culture. Cell lines were obtained from and cultured as per ATCC instructions. Cell lines were cultured in RPMI 1640 (K562) or DMEM (Hek293T, MDA-MB-231 and MDA-MB-468), 10\% foetal calf serum, 1\% penicillin/streptomycin (all Sigma-Aldrich Co. Ltd, Dorset, U.K.)

Serum profiling using protein arrays and ALL-patient sera. We performed antibody profiling on 13 samples from nine B-ALL patients, eight of which were prior to treatment, as well as nine age and sex-matched HV samples (Table 1). Each ProtoArray Human Protein Microarray v5.0 (Life Technologies) slide was immunoscreened with a single serum sample while one ProtoArrayR Control Microarray (Life Technologies) was used with control protein and one slide was assayed with secondary antibody alone. Signals from all peptides were analysed on the ProScanArray (PerkinElmer) using ProtoArray® Prospector v5.2 software (Life Technologies).

Prioritisation of $B-A L L$ antigens. To determine which genes warranted further investigation, we examined the antigens that were more frequently recognised by antibodies in sera from adult B-ALL than age- and sex-matched HV samples (at a $p$ value of $\leq 0.02$ ) using a systematic peer review process. We used Scopus and NCBI, and the search terms used were, where $\mathrm{X}$ represents each protein individually, ' $\mathrm{X}$ ', ' $\mathrm{X}$ AND cancer', 'X AND leukaemia', 'X AND 'acute lymphoblastic leukaemia', 'X AND function'; where available appropriate aliases were also searched in the same manner. In addition we performed STRING analysis (https://string-db.org/) to identify the processes these antigens engage in that are involved in cancer.

cDNA preparation from human samples. To evaluate the expression of the most promising antigens in $\mathrm{HV}$ and B-ALL cells, we isolated RNA from BM and peripheral blood (PB) samples using QIAGEN RNeasy ${ }^{\circledR}$ Plus mini kit (QIAGEN Ltd.) according to the manufacturer's protocol. cDNA was prepared using the MBI Fermentas RevertAid First Strand cDNA synthesis kit (MBI Fermentas Ltd, Helena BioSciences Ltd, Sunderland, U.K.) which was DNase I treated (Roche Products Ltd, Herts, U.K.), cleaned 
using a RNeasy kit (Qiagen), checked on a $1 \%$ agarose-TBE gel and quantified using a NanoDrop ${ }^{\mathrm{TM}}$ Lite Spectrophotometer (Thermo Scientific, UK).

Quantitative-Polymerase Chain Reaction ( $q P C R$ ) analysis. QPCR was performed using Quantinova $^{\mathrm{TM}} \mathrm{SYBR}^{\circledR}$ Green kit and QuantiTect Primers (all Qiagen). Glyceraldehyde 3-phosphate dehydrogenase $(G A P D H)$ and TATA-box binding protein $(T B P)$ were used as reference genes, chosen based on previous publications ${ }^{13}$ and in keeping with MIQE guidelines. ${ }^{14,15}$ Each primer was tested on at least one human cancer cell line that was known to express that antigen of interest (Table 2).

To control for gDNA contamination within the qPCR reagents, a no cDNA control was included on every qPCR plate whereby cDNA was replaced by RNase-free $\mathrm{H}_{2} \mathrm{O}$. In addition, each sample was analysed in triplicate on the 96-well qPCR plate (Applied Biosystems, USA). The reaction volumes were $10 \mu \mathrm{L} 2 \mathrm{X}$ QuantiNova SYBR green PCR Mix, $2 \mu \mathrm{L}$ QN ROX reference dye, $2 \mu \mathrm{L}$ QuantiTect primer assay and $5 \mu \mathrm{L}$ RNase-free $\mathrm{H}_{2} \mathrm{O}$, making a total volume of $19 \mu \mathrm{L}$ added to each well in the 96-well plate. In $1 \mu \mathrm{L}$ final volume, $\leq 100 \mathrm{ng}$ of template cDNA was added to achieve a final volume of $20 \mu 1$. The plate was then loaded into the thermocycler (StepOne Plus Real-Time PCR system, Applied Biosystems).

The cycle was 2 minutes at $95^{\circ} \mathrm{C}$, then 40 cycles of $5 \mathrm{~s}$ at $95^{\circ} \mathrm{C}$ followed by $10 \mathrm{~s}$ at $60^{\circ} \mathrm{C}$. This was immediately followed by a melt curve stage of $15 \mathrm{~s}$ at $95^{\circ} \mathrm{C}, 1 \mathrm{~min}$ at $60^{\circ} \mathrm{C}$ and $15 \mathrm{~s}$ at $95^{\circ} \mathrm{C}$, to verify the specificity of the amplification. Data was compared using StepOne software v2.0 (Applied Biosystems) and the comparative $\mathrm{C}_{\mathrm{T}}$ method. ${ }^{16} \mathrm{We}$ used the relative quantification (also comparative $\mathrm{C}_{\mathrm{T}}$ ) method to analyse the $\mathrm{qPCR}$ data.

Immunocytochemistry. Immunocytochemistry was performed as described previously ${ }^{17}$ using the antibodies detailed (Supplementary Table A1). Actin was used as a positive control for the successful performance of ICC while isotype, no primary and no secondary antibody immunolabelling acted as negative controls, used to detect nonspecific staining. Lillies-Mayer Haematoxylin was used as a counterstain.

Staining intensities were scored according to a five-tiered scale described originally by Biesterfeld et $\mathrm{al}^{18}$ as follows:- 0: no staining; 1: background; 2: weak staining; 3: moderate staining; 4: strong staining. The percentage of positively stained cells was 
based on the cell count of stained cells per microscopic view, and represented on a fivetiered scale $(0: 0 \% ; 1: 1-10 \% ; 2: 11-50 \% ; 3: 51-80 \% ; 4:>80 \%)$. The final immunoreactivity score was obtained by multiplication of the percentage of positive stained cells per microscopic view by the value for staining intensity $(0=$ negative, $1-29$ $=$ weak, $30-143=$ moderate, $144-228=$ high and $>228=$ very high staining) ${ }^{19}$

Microarray analysis of gene expression and clinical parameters. Finally we analysed a publically available microarray expression data (GSE38403) ${ }^{20}$ which encompassed 215 adult B-cell ALL and 12 pre-B samples $(\mathrm{CD} 19+$ and $\mathrm{VpreB}+)$ isolated from the bone marrows of HVs. For microarray analysis, the files were imported from GEO in the Partek Genomic Suite, normalised and subjected to ANOVA analysis.

\section{RESULTS}

Immunoprofiling of adult B-ALL patient sera using proto-arrays. The intensity values were used to undertake a per chip / per gene normalisation process and then ANOVA was performed on the 3,998 proteins, that had showed differences in recognition by $\mathrm{HV}$ and patient sera, across the 22 samples analysed (excluding the control). ANOVA analysis identified 69 significant genes that had a $p<0.05$ in terms of their recognition by ALL versus HV sera (Supplementary Table A2). The mean value and standard deviation of each signal was calculated to produce a z-score and showed that 19 proteins were more frequently recognised (Supplementary Table A2i) and 50 proteins were less frequently recognised by antibodies in B-ALL (Supplementary Table A2ii) compared with HV sera. Two proteins that had fold changes of less than 1.5 (POLK3K and RAB2IL1) were included as they were significantly different in their recognition by ALL versus HV sera. However, we focussed on antigens that were more frequently recognised by antibodies in B-ALL than HV sera because of our interest in identifying antigenic targets for immunotherapy.

Hierarchical cluster analysis was performed using Wards method (Figure 1A) and showed that B-ALL patients could be differentiated from HVs on the basis of protein recognition by sera. Principal component analysis (PCA)(Figure 1B) showed that apart from one HV sample, there was a good cluster distinction between the proteins 
recognised significantly more or less frequently by ALL compared with HV sera. Interestingly, the ALL cluster in the PCA plot is "tighter" than the HV cluster probably reflecting the homogeneity of the proteins uniquely recognised by B-ALL sera and the relative heterogeneity of the proteins recognised by antibodies in the HV samples.

Prioritisation of proto-array identified antigens. Through a systematic review of the literature, ${ }^{21}$ we identified bone marrow tyrosine kinase on chromosome X (BMX) also known as epithelial and endothelial tyrosine kinase (Ekt), dCTP pyrophosphatase 1 (DCTPP1) also known as XTP3-transactivated protein A (XTP3TPA) and vestigial like 4 (VGLL4) for further expression analysis. Each antigen was investigated by STRING analysis and shown to have pathways associated with subverted processes in cancer (Figure 2A). These three antigens were then analysed for their expression in samples from adult B-ALL and HVs by qPCR and ICC.

qPCR analysis of antigen expression in B-ALL and HV samples. We determined the relative expression of transcripts encoding the antigens of interest in the following cell lines that we used as controls (K562, HEK293T, MDA-MB-231 and MD-MB468)(Table 2). We included survivin in this study based on the promising results we had observed with this antigen, in terms of expression, in B-ALL compared with HV samples previously. ${ }^{22}$ All cell lines expressed all housekeeping genes as well as the four antigens under investigation except BMX which was not expressed in any of the cells lines, despite previous reports of its expression in Hek293T by Western Blotting. ${ }^{23}$

All samples from B-ALL patients and HVs, except for HV025, showed convincing GAPDH expression (Table 3) and all antigens tested (BMX, DCTTP1, Survivin and VGLL4) were expressed at lower levels than GAPDH, as expected. Survivin was expressed at higher levels than TBP in all cell lines, and DCTPP1 and VGLL4 were expressed at higher levels than TBP in K562 and Hek293T. TBP was recommended as a housekeeping gene for qPCR by other studies however we found TBP expression was not consistent between the samples. The differences between the HV samples were much smaller, after excluding HV025 from our analysis.

Patients ALL005 and ALL019 were not found to express detectable levels of any of the antigens studied (BMX, DCTPP1, survivin and VGLL4)(Figure 2B) while ALL020 expressed all four antigens. Of B-ALL patient samples analysed, two had detectable 
BMX expression (ALL003 and ALL020 PB), three patients expressed DCTPP1 (ALL002, ALL003 and ALL020 BM) and three patients expressed VGLL4 and survivin (ALL002, ALL003 and ALL020 PB and BM). ALL002 and ALL003 had also been examined by Boullosa et $\mathrm{al}^{22}$ and while ALL002 was found to have detectable survivin transcripts in both studies, ALL003 was negative for detectable survivin transcripts in this study. None of the HVs had consistently detectable levels of BMX, while all expressed DCTPP1 and VGLL4. In contrast to our previous findings, where four of five HVs did not express detectable levels of survivin, ${ }^{22}$ in this study transcripts were detected in three of four HVs (whom we had not analysed previously).

Immunolabelling of antigen expression in B-ALL using immunocytochemistry. We used actin as a positive control for immunolabelling and found it, as expected, to be present in all four control cell lines at high levels (Table 2), while the negative controls for this experiment (cells only, no primary antibody and isotype control) were all negative. HEK293T cell line was used to demonstrate we could immunolabel BMX and VGLL4, but only showed a moderate cytoplasmic expression of BMX and were negative for VGLL4; weak immunolabelling of survivin was also detected. The K562 cell line was used to demonstrate the immunolabelling of DCTPP1 and survivin, and showed moderate cytoplasmic levels of both. MDA-MB-231 cell line was used to demonstrate we could immunolabel DCTPP1, while MDA-MB-468 was chosen as an immunolabelling control for VGLL4 expression, but both were negative for the detection of these antigens. Of note VGLL4 was not found in any cell line, MDA-MB-231 and MDA-MB-468 did not express DCTPP1 or survivin, while each had transcripts from these antigens that were detectable by qPCR. In contrast BMX was detectable in Hek293T by ICC in agreement with previously published Western blotting data. ${ }^{23}$

Each antigen was found at moderate to high levels in patient samples (BMX - 4 of 6; DCTPP1 - 2 of 2; survivin - 1 of 2 and VGLL4 - 1 of 2) while each antigen was only found at low levels in HV samples (n=7)(Table 4; Figure 3). Of note ALL005 had also been examined for survivin expression in our previous paper ${ }^{22}$ and was found to be positive by ICC in this study also.

Gene expression microarray analysis. The antigens that were significantly more frequently recognised by B-ALL than HV sera $(p \leq 0.02)$ and were present in the 
microarray database (GSE38403) ${ }^{20}$ was examined for their association with above and below median levels of expression at disease diagnosis, and overall patient survival in the following 5 years post-diagnosis. Only cyclin G associated kinase (GAK) and high mobility group box family member 2 (TOX2) showed a significant association between above median expression at disease diagnosis and 5 -year overall survival $(p=0.024$ and $p=0.0056$ LogRank and $p=0.056$ and $p=0.028$ by Wilcoxon-Gehan, repectively; Figure 4A). When we analysed the levels of expression, segregated into quartiles, we found that only expression of TOX2 in the third quartile (Q3 - above median-low) was associated with 5 year survival while expression in the top quartile (Q4 - above median-high) was not (Figure 4B).

In addition a number of clinical features (stage and cytogenetic abnormalities) were found to be significantly associated with above or below median antigen expression at disease diagnosis (Supplementary Table A3). Of particular note was the high level of statistical significance $(p<0.001)$ for the following antigens and gender $(B M X)$, stage (APOBEC3A, DCTPP1, MUC20, SEPT9, TOX2 and WARS), early pre-B versus mature B-ALL (WARS), early pre-B versus early Pro-B (DCTPP1, MUC20, SEPT9, TOX2), early pre-B versus transitional Pre-B (VGLL4), mature B-ALL versus Pro-B (APOBEC3A), pre-B-ALL versus pro-B (MUC20), MLL mutation (DCTTP1, MUC20 and TOX2), BCR-ABL (DCTTP1, IGLL1, LMX1a, TOX2 and VGLL-4), other cytogenetic abnormalities (LMX1A), no cytogenetic abnormalities (IGLL1 and TOX2) and CD25 positivity (CDCA3, IGLL1, and TOX2).

\section{DISCUSSION}

Despite the recent development of a number of strategies for the treatment of adult BALL, survival rates remain low. This is mostly due to the toxicity of treatment to older adult B-ALL patients, the absence of B-ALL cell specific therapies, co-morbidities associated with the level of fitness of patients, treatment related toxicities and death due to disease recurrence. The circumvention of relapse due to improved treatments including matched unrelated stem cell transfusions, donor lymphocyte infusions and more recent 
immunotherapy strategies have had significant impacts on patient survival but they still have associated toxicity and antibody based therapies, especially, can be expensive. ${ }^{24}$ As part of the effort to identify new targets for immunotherapy, that can help delay if not prevent relapse after first remission, and to help us better understand the biological basis of adult B-ALL, we sero-profiled adult B-ALL patients and age and sex-matched HV sera. We identified 19 antigens that were significantly more frequently recognised by patient sera compared with HV sera and then prioritised these antigens based on literature searches. We identified three antigens that had been shown to play a significant role in cancer(s) and examined their expression in patient samples and HVs by qPCR in comparsion to survivin. We have previously shown that survivin has significantly higher expression in the B-ALL samples compared with healthy controls by qPCR analysis $(\mathrm{p}=0.015)$ and gene expression analysis $(p=0.013)^{22}$. However there was no statistically significant differences between the expression of the four antigens (BMX, DCTPP1, survivin or VGLL4) in patient samples compared with HVs by qPCR. Gene expression array analysis indicated that BMX, DCTPP1, survivin and VGLL4 transcript levels were each significantly decreased in B-ALL patients compared with healthy donors, while ICC analysis showed elevated protein levels in patients compared with healthy donors.

The discordance between detection of transcripts by qPCR and immunolabelling of antigens by ICC has been observed by scientists in other fields of research. It appears that there is a linear relationship between gene transcription and protein levels in Drosophila, ${ }^{58}$ that uncouples with evolutionary complexity, maximising in chimpanzees and humans ${ }^{59}$ and is partially explained by post-transcriptional regulation and differences in mRNA and protein turnover rates. Although $\mathrm{qPCR}$ is often used to semi-quantitate gene expression, we used gene expression arrays to examine transcript levels and found that protein levels, the ultimate target of immunotherapy strategies, are a more convincing indicator of the presence of an antigen in a cell. The finding of BMX, DCTPP1, survivin and VGLL4 at moderate levels in B-ALL samples and at low levels in HV samples, our systematic literature review (data not shown) and STRING analysis suggest that these proteins are worthy of further investigation as targets for the treatment of adult B-ALL. 
BMX was more frequently recognised by B-ALL patient compared to HV sera $(p=0.035)$ and is a particularly appealing target for immunotherapy, in accordance with the National Cancer Institute (NCI) priority list,${ }^{25}$ by virtue of it's location on the X chromosome. In addition, BMX has been shown to play a central role in the immune response, inflammation and cytokine signalling by regulating TNF-dependent signalling at a central node, that affects the efferent signalling branches of JNK, p38 MAPK and NK-kappa$\mathrm{B}^{26-28} \mathrm{BMX}$ has been reported to regulate multiple proteins ${ }^{27-31}$ and this was also apparent from the STRING analysis we performed. BMX is implicated in signal transduction pathways and the regulation of biological processes including apoptosis, cell migration, differentiation, proliferation and tumorigenesis. ${ }^{32-35}$ Aberrant BMX expression has been reported in a number of cancer types, including acute myeloid leukaemia,a and non-oncological diseases, such as rheumatoid arthritis, ischemia-mediated arteriogenesis and lymphangiogenesis. ${ }^{23,26-28,32,36-41}$

As a tyrosine kinase BMX is a a target for inhibitors including CI-1033, an epidermal growth factor receptor (EGFR) inhibitor, BMX-IN- $1^{44}$ and CTN06 ${ }^{45}$ a small molecule dual inhibitor of BMX and BTK, the latter shown to be effective in autophagy and apoptosis induction, as well as the inhibition of the growth and migration of prostate cancer cells. But it is the potential to target BMX by ibrutinib, an immunotherapy strategy that is being used with promise in mature B-cell malignancies including relapsed/refractory chronic lymphocytic leukaemia ${ }^{46-48}$, small lymphocytic lymphoma ${ }^{46}$, and mantle cell lymphoma ${ }^{49}$ that provides the most promise, especially in light of a recent study showing the ability of ibrutinib to target pre-BCR signalling in B-ALL. ${ }^{50}$

DCTPP1 acts as an intracellular regulator of 5-methyl-dCTP metabolism, which has been associated with DNA hypermethylation and gene silencing. ${ }^{51}$ This make DCTPP1 an interesting target for drug-resistant research, because of its association with DNA hypermethylation. DCTPP1 is downregulated when the PI3K/AKT/mTOR pathway is activated and autophagy activity is suppressed, and vice versa. ${ }^{52}$ Studies have shown the involvement of DCTPP1 in poor prognosis and lower overall survival of cancer patients, including breast cancer, gastric cancer, lung cancer, prostate cancer ${ }^{51-54}$ while DCTPP1 has also been shown to increase chemotherapy resistance in gastric cancer suggesting that it could potentially be an indicative biomarker of chemoresistance in patients. ${ }^{55}$ 
VGLL4 is a tumour suppressor gene located on 3p25.3-3p25.2 for further investigation because of it's demonstrated roles in proliferation, migration and cell death in a number of cancer types. ${ }^{56}$ VGLL4 is a co-factor for TEA domain-containing transcription factors (TEADs) $)^{57}$ and this association was re-itterated by our STRING analysis (Figure 2A).

It was also notable that WWOX appeared as one of the antigens that were less frequently recognised by patient than $\mathrm{HV}$ sera and this correlated with previous findings that WWOX was partially expressed or absent in over half of haematopoietic malignancies tested. $^{60,61}$ Indeed WWOX transcripts were reduced in ALL patient samples and methylation frequency increased when compared to healthy donors. ${ }^{62}$ Some debate remains as to whether WWOX is affected in leukaemias by virtue of it's location on 16q23.3-24.1 in FRA16D, a region of. However recent studies that have returned WWOX expression to leukaemia cells have shown repressed proliferation and increased rates of apoptosis. ${ }^{63}$

Due to the relative rarity of adult B-ALL ${ }^{64}$ the most effective way to determine expression of the antigens, we had identified, and their association with survival, stage and cytogenetics in a large cohort study, was to use a database of gene expression microarray analysis (GSE38403) ${ }^{20}$. We identified statistically significant correlations between the expression of 12 of the 19 antigens identified as more frequently recognised by adult B-ALL patients sera and a number of features of adult B-ALL disease. These features include gender, stage and cytogenetic abnormalities. Only two antigens, GAK and TOX2, had above median expression at disease diagnosis that significantly correlated with improved overall survival. We have seen similar results previously in acute myeloid leukaemia (AML) at disease presentation with SSX2IP, RHAMM and Survivin ${ }^{65}$ and reasoned that the elevated antigen expression could induce enhanced anti-tumour responses in patients following conventional treatment. We propose that as dead and dying leukaemia cells are mopped up by immune cells and their peptide contents presented on MHC in the context of 'danger' (inflammation) this could lead to more effective anti-tumour responses. However this isn't always the case with antigens such as PRAME which shows a Goldilocks effect in terms of both very high and very low expression being associated poor survival for myelodysplastic syndome patients, ${ }^{66}$ 
probably reflecting the impact of high PRAME expression on the tumourigenicity of the affected cells, and low levels enabling immune evasion following conventional treatment. In summary, we have used sero-profiling for the first time to characterise the recognition of antigens by antibodies in adult B-ALL sera, in direct comparison to age and sexmatched HV sera. We have identified new targets for treatment (BMX), provided new insights into the the biology underlying this rare and difficult to treat disease (loss of WWOX antigen recognition in adult B-ALL) and new biomarkers for survival (GAK, TOX2). 


\begin{abstract}
ABBREVIATIONS
B-ALL: B-cell acute lymphoblastic leukaemia; BMX: bone marrow tyrosine kinase on chromosome X; CAR: chimeric antigen receptor; DCTPP1: dCTP pyrophosphatase 1; GAK: cyclin G associated kinase; GAPDH: Glyceraldehyde 3-phosphate dehydrogenase; HV: healthy volunteer; PB: peripheral blood; PCA: Principal component analysis; qPCR: quantitative polymerase chain reaction; TBP: TATA-box binding protein; TEAD: TEA domain-containing transcription factors; TOX2: TOX high mobility group box family member 2 (TOX2) variant 3; VGLL4: vestigial like 4.
\end{abstract}

\title{
ACKNOWLEDGEMENTS
}

This work was supported by grants from Wessex Medical Research (B.G.) and British Society for Haematology (B.G. \& K.H.O); Erasmus exchange program (S.J.); Cancer Research U.K. Discovery Committee (S.B.) and Leukaemia \& Lymphoma NI (K.I.M.). S.J. and L.C. were MSc candidates at the University of Hull and this work was submitted in partial fulfilment of the requirement for the MSc.

CONTRIBUTION: S.J., and L.C.: designed and performed experiments, analysed results, made the figures and wrote the paper; S.B. and L.O., designed and performed experiments; K.I.M. analysed results, made the figures, contributed to writing of paper; K.H.O., designed experiments, wrote the paper; BG: designed and performed experiments, analysed results, made the figures and wrote the paper. Dr Mat Arno and the Genomics Centre, Kings College London; Dr Cindy Lee and Dr Hannah Wickenden for help and support.

Conflict-of-interest disclosure: The authors declare no competing financial interests

Correspondence: Dr Barbara Guinn, Department of Biomedical Sciences, Cottingham Road, University of Hull, Hull, HU7 6RX, UK; e-mail: B.Guinn@hull.ac.uk 


\section{REFERENCES}

1. Sant M, Minicozzi P, Mounier M, et al. Survival for haematological malignancies in Europe between 1997 and 2008 by region and age: results of EUROCARE-5, a population-based study. Lancet Oncol. 2014;15(9):931-942.

2. Fielding AK, Rowe JM, Richards SM, et al. Prospective outcome data on 267 unselected adult patients with Philadelphia chromosome-positive acute lymphoblastic leukemia confirms superiority of allogeneic transplantation over chemotherapy in the pre-imatinib era: results from the International ALL Trial MRC UKALLXII/ECOG2993. Blood. 2009;113(19):4489-4496.

3. Goldstone AH, Richards SM, Lazarus HM, et al. In adults with standard-risk acute lymphoblastic leukemia, the greatest benefit is achieved from a matched sibling allogeneic transplantation in first complete remission, and an autologous transplantation is less effective than conventional consolidation/maintenance chemotherapy in all patients: final results of the International ALL Trial (MRC UKALL XII/ECOG E2993). Blood. 2008;111(4):1827-1833.

4. Moorman AV, Harrison CJ, Buck GA, et al. Karyotype is an independent prognostic factor in adult acute lymphoblastic leukemia (ALL): analysis of cytogenetic data from patients treated on the Medical Research Council (MRC) UKALLXII/Eastern Cooperative Oncology Group (ECOG) 2993 trial. Blood. 2007;109(8):3189-3197.

5. Wei G, Wang J, Huang H, Zhao Y. Novel immunotherapies for adult patients with B-lineage acute lymphoblastic leukemia. J Hematol Oncol. 2017;10(1):150.

6. Pui $\mathrm{CH}$, Jeha S. New therapeutic strategies for the treatment of acute lymphoblastic leukaemia. Nat Rev Drug Discov. 2007;6(2):149-165.

7. Schwarzbich MA, Witzens-Harig M. Cellular Immunotherapy in B-Cell Malignancy. Oncol Res Treat. 2017;40(11):674-681.

8. Hathaway L, Sen JM, Keng M. Impact of blinatumomab on patient outcomes in relapsed/refractory acute lymphoblastic leukemia: evidence to date. Patient Relat Outcome Meas. 2018;9:329-337.

9. Loghavi S, Kutok JL, Jorgensen JL. B-acute lymphoblastic leukemia/lymphoblastic lymphoma. Am J Clin Pathol. 2015;144(3):393-410.

10. Bassan R, Bourquin JP, DeAngelo DJ, Chiaretti S. New Approaches to the Management of Adult Acute Lymphoblastic Leukemia. J Clin Oncol. 2018:JCO2017773648.

11. Gokbuget N. Treatment of Older Patients with Acute Lymphoblastic Leukaemia. Drugs Aging. 2018;35(1):11-26.

12. Horvat TZ, Seddon AN, Ogunniyi A, King AC, Buie LW, Daley RJ. The ABCs of Immunotherapy for Adult Patients With B-Cell Acute Lymphoblastic Leukemia. Ann Pharmacother. 2018;52(3):268-276.

13. Lossos IS, Czerwinski DK, Wechser MA, Levy R. Optimization of quantitative real-time RT-PCR parameters for the study of lymphoid malignancies. Leukemia. 2002;17(4):789-795.

14. Bustin SA, Benes V, Garson JA, et al. The MIQE guidelines: minimum information for publication of quantitative real-time PCR experiments. Clin Chem. 2009;55(4):611-622. 
15. Bustin SA, Wittwer CT. MIQE: A Step Toward More Robust and Reproducible Quantitative PCR. Clin Chem. 2017;63(9):1537-1538.

16. Livak KJ, Schmittgen TD. Analysis of relative gene expression data using realtime quantitative PCR and the 2(-Delta Delta C(T)) Method. Methods. 2001;25(4):402-408.

17. Khan G, Brooks SE, Mills KI, Guinn BA. Infrequent Expression of the CancerTestis Antigen, PASD1, in Ovarian Cancer. Biomark Cancer. 2015;7:31-38.

18. Biesterfeld S, Veuskens U, Schmitz FJ, Amo-Takyi B, Bocking A. Interobserver reproducibility of immunocytochemical estrogen- and progesterone receptor status assessment in breast cancer. Anticancer Res. 1996;16(5A):2497-2500.

19. Deng Z, Hasegawa M, Aoki K, et al. A comprehensive evaluation of human papillomavirus positive status and p16(INK4a) overexpression as a prognostic biomarker in head and neck squamous cell carcinoma. International Journal of Oncology. 2014;45(1):67-76.

20. Geng H, Brennan S, Milne TA, et al. Integrative epigenomic analysis identifies biomarkers and therapeutic targets in adult B-acute lymphoblastic leukemia. Cancer Discov. 2012;2(11):1004-1023.

21. Cooksey L. Identification and characterisation of novel antigens expressed by adult B-cell acute lymphocytic leukaemia cells at disease presentation $M S c$. 2018; University of Hull.

22. Boullosa LF, Savaliya P, Bonney S, et al. Identification of survivin as a promising target for the immunotherapy of adult B-cell acute lymphoblastic leukemia. Oncotarget. 2018;9(3):3853-3866.

23. van Oosterwijk JG, Buelow DR, Drenberg CD, et al. Hypoxia-induced upregulation of BMX kinase mediates therapeutic resistance in acute myeloid leukemia. J Clin Invest. 2018;128(1):369-380.

24. Hernandez I, Bott SW, Patel AS, et al. Pricing of monoclonal antibody therapies: higher if used for cancer? Am J Manag Care. 2018;24(2):109-112.

25. Cheever MA, Allison JP, Ferris AS, et al. The prioritization of cancer antigens: a national cancer institute pilot project for the acceleration of translational research. Clin Cancer Res. 2009;15(17):5323-5337.

26. Gottar-Guillier M, Dodeller F, Huesken D, et al. The tyrosine kinase BMX is an essential mediator of inflammatory arthritis in a kinase-independent manner. $J$ Immunol. 2011;186(10):6014-6023.

27. Holopainen T, Lopez-Alpuche V, Zheng W, et al. Deletion of the endothelial Bmx tyrosine kinase decreases tumor angiogenesis and growth. Cancer Res. 2012;72(14):3512-3521.

28. Li Y, Cui N, Zheng PS, Yang WT. BMX/Etk promotes cell proliferation and tumorigenicity of cervical cancer cells through PI3K/AKT/mTOR and STAT3 pathways. Oncotarget. 2017;8(30):49238-49252.

29. Saharinen P, Ekman N, Sarvas K, Parker P, Alitalo K, Silvennoinen O. The Bmx tyrosine kinase induces activation of the Stat signaling pathway, which is specifically inhibited by protein kinase Cdelta. Blood. 1997;90(11):4341-4353.

30. Chen S, Jiang X, Gewinner CA, et al. Tyrosine kinase BMX phosphorylates phosphotyrosine-primed motif mediating the activation of multiple receptor tyrosine kinases. Sci Signal. 2013;6(277):ra40. 
31. Wang Y, Xia J, Fang Z, et al. A novel BMX variant promotes tumor cell growth and migration in lung adenocarcinoma. Oncotarget. 2017;8(20):33405-33415.

32. Dai B, Chen H, Guo S, et al. Compensatory upregulation of tyrosine kinase Etk/BMX in response to androgen deprivation promotes castration-resistant growth of prostate cancer cells. Cancer Res. 2010;70(13):5587-5596.

33. Qiu Y, Kung HJ. Signaling network of the Btk family kinases. Oncogene. 2000;19(49):5651-5661.

34. Peng J, Wang Q, Liu H, Ye M, Wu X, Guo L. EPHA3 regulates the multidrug resistance of small cell lung cancer via the PI3K/BMX/STAT3 signaling pathway. Tumour Biol. 2016;37(9):11959-11971.

35. NCBI. BMX non-receptor tyrosine kinase [Homo sapiens]. https://www.ncbi.nlm.nih.gov/gene/660. Published 2019. Updated 13/02/2019. Accessed 01/03/2019.

36. Qiu Y, Robinson D, Pretlow TG, Kung HJ. Etk/Bmx, a tyrosine kinase with a pleckstrin-homology domain, is an effector of phosphatidylinositol 3'-kinase and is involved in interleukin 6-induced neuroendocrine differentiation of prostate cancer cells. Proc Natl Acad Sci U S A. 1998;95(7):3644-3649.

37. Guo S, Sun F, Guo Z, et al. Tyrosine kinase ETK/BMX is up-regulated in bladder cancer and predicts poor prognosis in patients with cystectomy. PLoS One. 2011;6(3): e17778.

38. Zhuang J, Tu X, Cao K, et al. The expression and role of tyrosine kinase ETK/BMX in renal cell carcinoma. J Exp Clin Cancer Res. 2014;33:25.

39. Potter DS, Galvin M, Brown S, et al. Inhibition of PI3K/BMX Cell Survival Pathway Sensitizes to BH3 Mimetics in SCLC. Mol Cancer Ther. 2016;15(6):1248-1260.

40. Eldeeb MA, Fahlman RP. Phosphorylation Impacts N-end Rule Degradation of the Proteolytically Activated Form of BMX Kinase. J Biol Chem. 2016;291(43):22757-22768.

41. Wei T, Zhu W, Fang S, et al. miR-495 promotes the chemoresistance of SCLC through the epithelial-mesenchymal transition via Etk/BMX. Am J Cancer Res. 2017;7(3):628-646.

42. Jarboe JS, Dutta S, Velu SE, Willey CD. Mini-review: bmx kinase inhibitors for cancer therapy. Recent Pat Anticancer Drug Discov. 2013;8(3):228-238.

43. Hur W, Velentza A, Kim S, et al. Clinical stage EGFR inhibitors irreversibly alkylate Bmx kinase. Bioorg Med Chem Lett. 2008;18(22):5916-5919.

44. Liu F, Zhang X, Weisberg E, et al. Discovery of a selective irreversible BMX inhibitor for prostate cancer. ACS Chem Biol. 2013;8(7):1423-1428.

45. Guo W, Liu R, Bhardwaj G, et al. Targeting Btk/Etk of prostate cancer cells by a novel dual inhibitor. Cell Death Dis. 2014;5:e1409.

46. Coutre SE, Byrd JC, Hillmen P, et al. Long-term safety of single-agent ibrutinib in patients with chronic lymphocytic leukemia in 3 pivotal studies. Blood Adv. 2019;3(12):1799-1807.

47. Nuttall E, Tung J, Trounce E, Johnston R, Chevassut T. Real-world experience of ibrutinib therapy in relapsed chronic lymphocytic leukemia: results of a singlecenter retrospective analysis. J Blood Med. 2019;10:199-208. 
48. Shanafelt TD, Wang XV, Kay NE, et al. Ibrutinib-Rituximab or Chemoimmunotherapy for Chronic Lymphocytic Leukemia. $N$ Engl J Med. 2019;381(5):432-443.

49. Wang ML, Rule S, Martin P, et al. Targeting BTK with ibrutinib in relapsed or refractory mantle-cell lymphoma. $N$ Engl J Med. 2013;369(6):507-516.

50. Kim E, Hurtz C, Koehrer S, et al. Ibrutinib inhibits pre-BCR $(+)$ B-cell acute lymphoblastic leukemia progression by targeting BTK and BLK. Blood. 2017;129(9):1155-1165.

51. Song FF, Xia LL, Ji P, et al. Human dCTP pyrophosphatase 1 promotes breast cancer cell growth and stemness through the modulation on 5-methyl-dCTP metabolism and global hypomethylation. Oncogenesis. 2015;4:e159.

52. Lu J, Dong W, He H, et al. Autophagy induced by overexpression of DCTPP1 promotes tumor progression and predicts poor clinical outcome in prostate cancer. Int J Biol Macromol. 2018;118(Pt A):599-609.

53. Zhang Y, Ye WY, Wang JQ, et al. dCTP pyrophosphohydrase exhibits nucleic accumulation in multiple carcinomas. Eur J Histochem. 2013;57(3):e29.

54. Morisaki T, Yashiro M, Kakehashi A, et al. Comparative proteomics analysis of gastric cancer stem cells. PLoS One. 2014;9(11):e110736.

55. Xia LL, Tang YB, Song FF, et al. DCTPP1 attenuates the sensitivity of human gastric cancer cells to 5-fluorouracil by up-regulating MDR1 expression epigenetically. Oncotarget. 2016;7(42):68623-68637.

56. Deng X, Fang L. VGLL4 is a transcriptional cofactor acting as a novel tumor suppressor via interacting with TEADs. Am J Cancer Res. 2018;8(6):932-943.

57. Zhang Y, Shen H, Withers HG, et al. VGLL4 Selectively Represses YAPDependent Gene Induction and Tumorigenic Phenotypes in Breast Cancer. Sci Rep. 2017;7(1):6190.

58. Lemos B, Bettencourt BR, Meiklejohn CD, Hartl DL. Evolution of proteins and gene expression levels are coupled in Drosophila and are independently associated with mRNA abundance, protein length, and number of protein-protein interactions. Mol Biol Evol. 2005;22(5):1345-1354.

59. Fu N, Drinnenberg I, Kelso J, et al. Comparison of protein and mRNA expression evolution in humans and chimpanzees. PLoS One. 2007;2(2):e216.

60. Ishii H, Vecchione A, Furukawa Y, et al. Expression of FRA16D/WWOX and FRA3B/FHIT genes in hematopoietic malignancies. Mol Cancer Res. 2003;1(13):940-947.

61. Cui Z, Lin D, Cheng F, et al. The role of the WWOX gene in leukemia and its mechanisms of action. Oncol Rep. 2013;29(6):2154-2162.

62. Chen X, Zhang H, Li P, Yang Z, Qin L, Mo W. Gene expression of WWOX, FHIT and p73 in acute lymphoblastic leukemia. Oncol Lett. 2013;6(4):963-969.

63. Lin D, Cui Z, Kong L, Cheng F, Xu J, Lan F. p73 participates in WWOXmediated apoptosis in leukemia cells. Int J Mol Med. 2013;31(4):849-854.

64. Inaba H, Greaves M, Mullighan CG. Acute lymphoblastic leukaemia. Lancet. 2013;381(9881):1943-1955.

65. Guinn B, Greiner J, Schmitt M, Mills KI. Elevated expression of the leukemiaassociated antigen SSX2IP predicts survival in acute myeloid leukemia patients who lack detectable cytogenetic rearrangements. Blood. 2009;113(5):1203-1204. 
66. Liberante FG, Pellagatti A, Boncheva V, et al. High and low, but not intermediate, PRAME expression levels are poor prognostic markers in myelodysplastic syndrome at disease presentation. Br J Haematol. 2013;162(2):282-285. 
